\title{
Bribe-proof Rules in the Division Problem*
}

\author{
Jordi Massó ${ }^{\dagger}$ and Alejandro Neme ${ }^{\ddagger}$
}

\section{October 2006}

\begin{abstract}
The division problem consists of allocating an amount of a perfectly divisible good among a group of $n$ agents with single-peaked preferences. A rule maps preference profiles into $n$ shares of the amount to be allocated. A rule is bribe-proof if no group of agents can compensate one of its subgroups to misrepresent their preferences and, after an appropriate redistribution of their shares, each obtain a weakly preferred share and all agents in the misrepresenting subgroup obtain a strictly preferred share. We characterize all bribe-proof rules as the class of Pareto efficient, strategy-proof, and weakly replacement monotonic rules. This class is larger than the set of sequential allotment rules identified in Barberà, Jackson, and Neme (1997).

Keywords: Bribe-proofness, Strategy-proofness, Pareto Efficiency, Replacement Monotonicity, Single-peakedness.

Journal of Economic Literature Classification Numbers: D71, D78, D63.
\end{abstract}

*We thank Dolors Berga, Lars Ehlers, Flip Klijn, an Associate Editor, and two referees for helpful comments and suggestions. The work of A. Neme is partially supported by the Universidad Nacional de San Luis through grant 319502, by the Consejo Nacional de Investigaciones Científicas y Técnicas CONICET, through grant PICT-02114, and by the Agencia Nacional de Promoción Científica y Técnica, through grant 03-10814. The work of Jordi Massó is partially supported by the grant SEJ2005-01481/ECON and FEDER from the Spanish Ministerio de Ciencia y Tecnología, and grant SGR2005-00454 and the Barcelona Economics Program (XREA) from the Generalitat de Catalunya. J. Massó and A. Neme acknowledge financial support from a grant PCI España-Iberoamérica 2005 (Programa de Cooperación Interuniversitaria de la Agencia Española de Cooperación Internacional-AECI).

†Departament d'Economia i d'Història Econòmica and CODE. Universitat Autònoma de Barcelona. 08193, Bellaterra (Barcelona), Spain. E-mail: jordi.masso@uab.es

${ }^{\ddagger}$ Instituto de Matemática Aplicada de San Luis. Universidad Nacional de San Luis and CONICET. Ejército de los Andes 950. 5700, San Luis, Argentina. E-mail: aneme@unsl.edu.ar 


\section{Introduction}

This paper investigates the set of bribe-proof rules in the context of the division problem. The division problem consists of allocating a fixed amount of a perfectly divisible good (or a task) among a group of $n$ agents. A rule maps preference profiles into $n$ shares of the amount to be allocated. This is often considered as an instance of an economy with private goods since an allocation is a $n$-dimensional vector (specifying the amount allocated to each agent) and each agent only cares about his own share. ${ }^{1}$ Sprumont (1991) shows that if each agent has single-peaked preferences over his share, then the uniform rule is the unique Pareto efficient, strategy-proof, and anonymous rule. A preference relation is single-peaked if it has a unique maximal share and on each of its sides the preference relation is strictly decreasing. Single-peakedness is a meaningful domain restriction if we interpret the division problem as the reduced model of a more general problem where the good has a fixed price (or the task is paid at a fixed wage); then, strictly increasing and convex preferences in the two-dimensional space defined by the share of the good (or effort on the task) and money will generate single-peaked preferences on the interval of all possible shares. Strategy-proofness requires that no agent can obtain a better share by misrepresenting his preference relation. Pareto efficiency requires that no group of agents can obtain better shares by redistributing their original shares. In the context of an allocation problem where there is a perfectly transferable good it is natural to combine both principles and to require that rules should be bribeproof in the sense that no group of agents can compensate one of its subgroups to misrepresent their preferences and, after an appropriate redistribution of their shares, each obtain a weakly preferred share and all agents in the misrepresenting subgroup obtain a strictly preferred share.

Bribe-proofness combines the principles of Pareto efficiency and strategy-proofness. It is very well understood that in public goods economies Pareto efficiency and strategy-proofness are incompatible unless social choice functions are either dictatorial or restricted to operate in small preference domains. ${ }^{2}$ In contrast, Barberà, Jackson, and Neme (1997) shows that the class of Pareto efficient and strategy-

\footnotetext{
${ }^{1}$ Sprumont (1995), Barberà (1996), Barberà (2001), and Barberà (2006) are surveys of the literature on strategy-proofness in which the division problem plays a distinguished role.

${ }^{2}$ For instance, in the context of a society choosing a level of a public good, Moulin (1980) characterizes Pareto efficient and strategy-proof social choice functions as a particular subclass of generalized median voter schemes whenever agents have single-peaked preference relations on the set of possible levels of the public good and monetary transfers among agents are not admissible.
} 
proof rules in the division problem is very large. Specifically, they characterize the set of sequential allotment rules as the class of Pareto efficient, strategy-proof, and replacement monotonic rules. Replacement monotonicity requires that if an agent receives a larger share after changing his preference relation then all the other agents should receive smaller shares.

Schummer (2000) proposes, for general economies with quasi-linear utilities, a similar concept of bribe-proofness. Roughly, Schummer (2000) shows that under a bribe-proof rule, each agent's payoff is continuous with respect to other agents' reported valuations and identifies conditions on the domain of valuations under which bribe-proof rules must be constant. In addition to Schummer (2000), very few papers have studied bribe-proofness. Esö and Schummer (2004) examines whether a second-price auction with two bidders (with private and independent values) is bribe-proof in the sense that a bidder may pay the other bidder to submit a zero bid. In the context of the marriage model with endowments Fiestras-Janeiro, Klijn, and Sánchez (2004) characterizes the class of consumption rules under which optimal rules are immune to manipulation via predonation of endowments.

The results of the paper and its organization are the following. After notation and basic definitions, Section 2 presents the properties of Pareto efficiency, strategyproofness, and bribe-proofness as well as some of the basic relationships among them. In particular, Lemma 1 states that all bribe-proof rules are Pareto efficient and strategy-proof and Example 1 exhibits a Pareto efficient and strategy-proof rule that is not bribe-proof. Section 3 introduces the properties of replacement monotonicity and weak replacement monotonicity. Examples 2 and 3 show that the class of all bribe-proof rules is strictly larger than the set of Pareto efficient, strategy-proof, and replacement monotonic rules. The main result of the paper (Theorem 1) states that a rule is bribe-proof if and only if it is Pareto efficient, strategy-proof, and weakly replacement monotonic. This implies that the class of all bribe-proof rules is strictly larger than the family of sequential allotment rules identified in Barberà, Jackson, and Neme (1997). Section 4 contains the proof of Theorem 1. Section 5 concludes by first showing the consequences of restricting in the definition of bribe-proofness (as in Schummer (2000)) the sets of bribers and bribed agents to be both singleton sets. Second, we discuss the consequences of considering a stronger definition of bribe-proofness by allowing that each bribed agent be indifferent between his original share and the share received once he is compensated after declaring another preference relation. In particular, we show that the uniform rule would not be bribe-proof according to this stronger definition. 
Finally, we indicate how to weaken the definition of sequential allotment rule (see Barberà, Jackson, and Neme (1997)) to obtain the set of weakly sequential rules, which coincides with the class of all bribe-proof and tops-only rules.

\section{Preliminaries}

Agents are the elements of a finite set $N=\{1, \ldots, n\}$ where $n \geq 2$. They have to share a given amount of a perfectly divisible good that, without loss of generality, we assume to be equal to 1 . An allocation is a vector $\left(x_{1}, \ldots, x_{n}\right) \in \mathbb{R}_{+}^{n}$ such that $\sum_{i \in N} x_{i}=1$. We denote by $A$ the set of allocations. Each agent $i \in N$ has a complete preorder $R_{i}$ over $[0,1]$, his preference relation. Let $P_{i}$ be the strict preference relation associated with $R_{i}$ and let $I_{i}$ be its indifference relation. We assume that agents have continuous preference relations in the sense that for each $x \in[0,1]$ the sets $\left\{y \in[0,1] \mid x R_{i} y\right\}$ and $\left\{y \in[0,1] \mid y R_{i} x\right\}$ are closed. Preference relations are assumed to be single-peaked. That is, $R_{i}$ is single-peaked if it has a unique maximal share $\tau\left(R_{i}\right) \in[0,1]$, the top of $R_{i}$, such that for any $x, y \in[0,1]$, we have $x P_{i} y$ whenever $y<x<\tau\left(R_{i}\right)$ or $\tau\left(R_{i}\right)<x<y$.

We denote by $\mathcal{R}$ the set of continuous and single-peaked preference relations on $[0,1]$. Preference profiles are $n$-tuples of preference relations on $[0,1]$ and they are denoted by $R=\left(R_{1}, \ldots, R_{n}\right) \in \mathcal{R}^{N}$. When we want to stress the role of agent $i$ 's preference relation we will represent a preference profile $R$ by $\left(R_{i}, R_{-i}\right)$ and by $\left(R_{S}, R_{-S}\right)$ if we want to stress the role in $R$ of the preference relations of agents in $S$.

A rule is a function $\Phi: \mathcal{R}^{N} \longrightarrow A$; that is, $\sum_{i \in N} \Phi_{i}(R)=1$ for all $R \in \mathcal{R}^{N}$.

A minimal requirement on rules is Pareto efficiency. Given a preference profile $R \in \mathcal{R}^{N}$, an allocation $x \in A$ is Pareto efficient if there is no $z \in A$ such that for all $i \in N, z_{i} R_{i} x_{i}$, and for at least one $j \in N$ we have $z_{j} P_{j} x_{j}$. Denote by $E(R)$ the set of Pareto efficient allocations.

A rule is Pareto efficient if it always selects a Pareto efficient allocation. Formally,

Definition 1 A rule $\Phi$ is Pareto efficient if for all $R \in \mathcal{R}^{N}, \Phi(R) \in E(R)$.

It is immediate to verify that, when preference profiles are single-peaked, Pareto efficient rules ration out all agents in the same side of their tops. That is, for each $R \in \mathcal{R}^{N}:$ 


$$
\left[\sum_{j \in N} \tau\left(R_{j}\right) \geq 1\right] \Longrightarrow\left[\tau\left(R_{i}\right) \geq \Phi_{i}(R) \text { for all } i \in N\right]
$$

and

$$
\left[\sum_{j \in N} \tau\left(R_{j}\right)<1\right] \Longrightarrow\left[\tau\left(R_{i}\right) \leq \Phi_{i}(R) \text { for all } i \in N\right] .
$$

Rules require each agent to report a preference relation. A rule is strategyproof if it is always in the best interest of an agent to reveal his preference relation truthfully. Formally,

Definition 2 A rule $\Phi$ is strategy-proof if for all $R \in \mathcal{R}^{N}$, all $i \in N$, and all $R_{i}^{\prime} \in \mathcal{R}, \Phi_{i}\left(R_{i}, R_{-i}\right) R_{i} \Phi_{i}\left(R_{i}^{\prime}, R_{-i}\right)$.

In addition to Pareto efficiency and strategy-proofness we are specially interested in rules that preclude the possibility that a group of agents gain by reallocating their shares after one of its subgroups misrepresent their preference relations. Formally,

Definition $3 A$ rule $\Phi$ is bribe-proof if for all $R \in \mathcal{R}^{N}$ and all $T \subseteq S \subseteq N$, there are no $R_{T}^{\prime} \equiv\left(R_{j}^{\prime}\right)_{j \in T} \in \mathcal{R}^{T}$ and $\left(t_{j}\right)_{j \in S} \in \mathbb{R}^{S}$ such that $\sum_{j \in S} t_{j}=\sum_{j \in S} \Phi_{j}\left(R_{T}^{\prime}, R_{-T}\right)$, $t_{j} R_{j} \Phi_{j}(R)$ for all $j \in S$, and $t_{i} P_{i} \Phi_{i}(R)$ for all $i \in T$.

We will refer to agents in $S \backslash T$ as bribers and to agents in $T$ as bribed agents. A few comments about the notion of bribe-proofness are appropriated. First, observe that only bribed agents jointly misrepresent their preference relations although, after reallocating their new shares, bribers weakly gain while all bribed agents strictly gain. Second, the cardinalities of $S$ and $T$ are only restricted to satisfy $\# T \leq \# S \leq n$. Schummer's (2000) definition of bribe-proofness requires $\# T \leq \# S \leq 2$. Along the proof of Theorem 1 (Remark 2 and Lemma 5), we will show that our notion of bribe-proofness coincides with the notion of individually bribe-proofness in which it is required that $\# T \leq 1$ and $\# T \leq \# S \leq n$. Third, as in Schummer (2000), one could require that all agents, bribers as well as bribed agents, be strictly better off after the misrepresentation of the preference relations and the reallocation of the shares. Our Theorem 1 remains unchanged with this alternative definition (see Massó and Neme (2003)). However, as we will argue at the end of the paper, the requirement that all bribed agents should be strictly better off is crucial; otherwise, if bribed agents may remain indifferent, then bribe-proofness becomes too stringent and even the uniform rule is not bribe-proof according to 
this stronger notion. ${ }^{3}$ Finally, our notion of bribe-proofness implicitly assumes two things. First, after the misrepresentation bribers and bribed agents can enforce the payment of bribes; thus, and since the arrangement of the reallocation of shares depends on the agents' true preference relations, agents in $S$ must trust each other. Second, the deviating coalition $S$ does not worry about a further bribe in response to the first one. These two issues potentially undermine the bite of bribe-proofness and they deserve further research that is left for the future. However, and following Schummer's (2000) similar discussion on the first issue, a notion that accommodates these two conceptual difficulties would make bribes more difficult; thus, rules that are bribe-proof according to our definition will remain bribe-proof after taking into account these considerations.

Our first preliminary result establishes that all bribe-proof rules are indeed Pareto efficient and strategy-proof.

Lemma 1 All bribe-proof rules are Pareto efficient and strategy-proof.

Proof The fact that a bribe-proof rule is strategy-proof follows immediately after considering in the definition of bribe-proofness the particular case where $S=$ $T=\{i\}$. Assume $\Phi$ is not Pareto efficient; that is, there exist $R \in \mathcal{R}^{N}, i \in$ $N$, and $\left(x_{1}, \ldots, x_{n}\right) \in A$ such that $x_{j} R_{j} \Phi_{j}(R)$ for all $j \in N$ and $x_{i} P_{i} \Phi_{i}(R)$. Take $Q=\left\{k \in N \mid x_{k} \neq \Phi_{k}(R)\right\}$. Define $Q_{+}=\left\{k \in N \mid x_{k}>\Phi_{k}(R)\right\}$ and $Q_{-}=$ $\left\{k \in N \mid x_{k}<\Phi_{k}(R)\right\}$. Observe that $Q=Q_{+} \cup Q_{-}$and $i \in Q$. Without loss of generality assume that $i \in Q_{+}$. Then, there exists at least one agent $j \in Q_{-}$. Since $R_{i}$ and $R_{j}$ are single-peaked, $\tau\left(R_{i}\right)>\Phi_{i}(R)$ and $\tau\left(R_{j}\right)<\Phi_{j}(R)$. Therefore, there exists $\varepsilon>0$ sufficiently small such that $\tau\left(R_{i}\right)>\Phi_{i}(R)+\varepsilon$ and $\tau\left(R_{j}\right)<\Phi_{j}(R)-\varepsilon$. Define $S=\{i, j\}, T=\{i\}, R_{i}^{\prime}=R_{i}, t_{i}=\Phi_{i}(R)+\varepsilon$, and $t_{j}=\Phi_{j}(R)-\varepsilon$. Notice that $t_{i}+t_{j}=\Phi_{i}(R)+\Phi_{j}(R), t_{i} P_{i} \Phi_{i}(R)$, and $t_{j} P_{j} \Phi_{j}(R)$. Hence, $\Phi$ is not bribe-proof.

Example 1 below exhibits a Pareto efficient and strategy-proof rule that is not bribe-proof.

Example 1 Let $N=\{1,2,3\}$ be the set of agents and define the rule $\phi: \mathcal{R}^{N} \rightarrow A$

\footnotetext{
${ }^{3}$ The uniform rule tries to allocate the shares as equal as possible, keeping the bounds imposed by Pareto efficiency (see Sprumont (1991) for its description and characterizations).
} 
as follows. For all $R \in \mathcal{R}^{N}$,

$$
\begin{aligned}
& \phi_{1}(R)=\max \left\{0,1-\tau\left(R_{2}\right)-\tau\left(R_{3}\right)\right\}, \\
& \phi_{2}(R)= \begin{cases}\tau\left(R_{2}\right) & \text { if } 0 R_{1} 1 \\
\min \left\{\tau\left(R_{2}\right), 1-\tau\left(R_{3}\right)\right\} & \text { if } 1 P_{1} 0,\end{cases} \\
& \phi_{3}(R)= \begin{cases}\tau\left(R_{3}\right) & \text { if } 1 P_{1} 0 \\
\min \left\{\tau\left(R_{3}\right), 1-\tau\left(R_{2}\right)\right\} & \text { if } 0 R_{1} 1 .\end{cases}
\end{aligned}
$$

Observe that $\phi$ is Pareto efficient and strategy-proof. To see that $\phi$ is not bribeproof, consider any $R \in \mathcal{R}^{N}$ such that $\tau\left(R_{1}\right)=\frac{1}{2}, 0 P_{1} 1$, and $\tau\left(R_{2}\right)=\tau\left(R_{3}\right)=1$. Then $\phi(R)=(0,1,0)$. Let $R_{1}^{\prime} \in \mathcal{R}$ be such that $\tau\left(R_{1}^{\prime}\right)=\frac{1}{2}$ and $1 P_{1}^{\prime} 0$. Then, $\phi\left(R_{1}^{\prime}, R_{-1}\right)=(0,0,1)$. Consider $S=\{1,3\}, T=\{1\}$, and $t_{1}=t_{3}=\frac{1}{2}$. Since $\frac{1}{2} P_{1} 0$ and $\frac{1}{2} P_{3} 0$, $\phi$ is not bribe-proof.

Lemmata 1 and 2 in Sprumont (1991) provides a fundamental one-agent result about strategy-proof rules. The following Lemma, which will be intensively used henceforth, adapts Sprumont's (1991) result to rules with more than one agent.

Lemma 2 Let $\Phi$ be a Pareto efficient and strategy-proof rule. Then, for every $i \in N$ and $R \in \mathcal{R}^{N}$ there exist $0 \leq a\left(R_{-i}\right) \leq b\left(R_{-i}\right) \leq 1$ such that $\Phi_{i}\left(R_{i}, R_{-i}\right)=$ med $\left\{a\left(R_{-i}\right), b\left(R_{-i}\right), \tau\left(R_{i}\right)\right\} .{ }^{4}$

\section{An Axiomatic Characterization of All Bribe- proof Rules}

Barberà, Jackson, and Neme (1997) characterizes the class of Pareto efficient and strategy-proof rules that satisfy the following additional requirement of monotonicity.

Definition 4 A rule $\Phi$ is replacement monotonic if for all $R \in \mathcal{R}^{N}$, all $i \in N$, and all $R_{i}^{\prime} \in \mathcal{R}$, if $\left[\Phi_{i}(R) \leq \Phi_{i}\left(R_{i}^{\prime}, R_{-i}\right)\right]$ then $\left[\Phi_{j}(R) \geq \Phi_{j}\left(R_{i}^{\prime}, R_{-i}\right)\right.$ for all $\left.j \neq i\right]$.

Replacement monotonicity requires a symmetric treatment of agents. An increase in agent $i$ 's share after $i$ changes his preference relation decreases the total amount left to be allocated among the remaining agents. Replacement monotonicity implies that none of the remaining agents' shares can increase.

\footnotetext{
${ }^{4}$ Given $x, y, z \in[0,1]$, not necessarily different, define med $\{x, y, z\}$ as the number $w \in\{x, y, z\}$ such that $\#\left\{w^{\prime} \in\{x, y, z\} \mid w^{\prime} \geq w\right\} \geq 2$ and $\#\left\{w^{\prime} \in\{x, y, z\} \mid w^{\prime} \leq w\right\} \geq 2$.
} 
Proposition 1 All Pareto efficient, strategy-proof, and replacement monotonic rules are bribe-proof.

Proposition 1 will immediately follow from Remark 1 and Theorem 1 below and therefore its proof is omitted.

Example 2 below illustrates the fact that Pareto efficiency, strategy-proofness, and replacement monotonicity do not fully characterize the class of bribe-proof rules since $\psi$ is bribe-proof (and, by Lemma 1, Pareto efficient and strategy-proof) but it is not replacement monotonic.

Example 2 Let $N=\{1,2,3\}$ be the set of agents and define the rule $\psi: \mathcal{R}^{N} \rightarrow A$ as follows. For all $R \in \mathcal{R}^{N}$,

$$
\begin{aligned}
& \psi_{1}(R)=\tau\left(R_{1}\right) \\
& \psi_{2}(R)= \begin{cases}\min \left\{\tau\left(R_{2}\right), 1-\tau\left(R_{1}\right)\right\} & \text { if } 0 R_{1} 1 \\
\max \left\{0,1-\tau\left(R_{1}\right)-\tau\left(R_{3}\right)\right\} & \text { if } 1 P_{1} 0,\end{cases} \\
& \psi_{3}(R)= \begin{cases}\min \left\{\tau\left(R_{3}\right), 1-\tau\left(R_{1}\right)\right\} & \text { if } 1 P_{1} 0 \\
\max \left\{0,1-\tau\left(R_{1}\right)-\tau\left(R_{2}\right)\right\} & \text { if } 0 R_{1} 1 .\end{cases}
\end{aligned}
$$

To show that $\psi$ is bribe-proof assume otherwise and let $T \subseteq S$ be the corresponding sets of agents involved in the bribe at $R \in \mathcal{R}^{N}$. Since $\psi$ rations out all agents in the same side of their tops, $\psi$ is Pareto efficient. Moreover, no agent can manipulate $\psi$ because all agents either receive their top or are only able to change their shares in the opposite direction of their top. Thus, $\# S \neq 1$ and $\# S \neq 3$. Observe that for all $\hat{R} \in \mathcal{R}^{N}, \psi_{1}(\hat{R})=\tau\left(\hat{R}_{1}\right)$. Hence, $1 \notin T$ and, without loss of generality, assume that $1 \in S$. Thus, $S=\{1, j\}$ and $T=\{j\}$, where $j$ is equal to either 2 or 3 . Let $\left(t_{1}, t_{j}\right) \in \mathbb{R}^{\{1, j\}}$ and $R_{j}^{\prime} \in \mathcal{R}$ be such that $t_{1}+t_{j}=\psi_{1}\left(R_{j}^{\prime}, R_{-j}\right)+\psi_{j}\left(R_{j}^{\prime}, R_{-j}\right)$. Since $\psi_{1}\left(R_{j}^{\prime}, R_{-j}\right)=\tau\left(R_{1}\right)=\psi_{1}(R), \psi_{j}\left(R_{j}^{\prime}, R_{-j}\right)=t_{j} . \quad$ By hypothesis, $t_{j}=$ $\psi_{j}\left(R_{j}^{\prime}, R_{-j}\right) P_{j} \psi_{j}(R)$, implying that $\psi$ is not strategy-proof, a contradiction. Hence, $\psi$ is bribe-proof. Observe that $\psi$ is not replacement monotonic. To see it, consider any $\left(R_{1}, R_{2}, R_{3}\right) \in \mathcal{R}^{N}$ and $R_{1}^{\prime} \in \mathcal{R}$ such that $\left(\tau\left(R_{1}\right), \tau\left(R_{2}\right), \tau\left(R_{3}\right)\right)=\left(\frac{1}{4}, 1,1\right)$, $0 P_{1} 1, \tau\left(R_{1}^{\prime}\right)=\frac{3}{4}$, and $1 P_{1}^{\prime} 0$. Then, $\psi\left(R_{1}, R_{2}, R_{3}\right)=\left(\frac{1}{4}, \frac{3}{4}, 0\right)$ and $\psi\left(R_{1}^{\prime}, R_{2}, R_{3}\right)=$ $\left(\frac{3}{4}, 0, \frac{1}{4}\right)$, which means that $\psi$ is not replacement monotonic.

Tops-only rules are specially interesting because they have the informationally nice feature that they only require agents to reveal their best-shares since they only depend on their tops. Formally, a rule $\Phi$ is tops-only if for all $R, R^{\prime} \in \mathcal{R}^{N}$ such that 
$\tau\left(R_{i}\right)=\tau\left(R_{i}^{\prime}\right)$ for all $i \in N, \Phi(R)=\Phi\left(R^{\prime}\right)$. Observe that the rule $\psi$ in Example 2 is not tops-only. Example 3 below shows that the equivalence between bribe-proof rules and Pareto efficient, strategy-proof, and replacement monotonic rules does not hold even in the subclass of tops-only rules.

Example 3 Let $N=\{1,2,3\}$ be the set of agents and define $\Psi: \mathcal{R}^{N} \rightarrow A$ as follows. For all $R \in \mathcal{R}^{N}$,

$$
\begin{aligned}
& \Psi_{1}(R)=\tau\left(R_{1}\right), \\
& \Psi_{2}(R)= \begin{cases}\min \left\{\tau\left(R_{2}\right), 1-\tau\left(R_{1}\right)\right\} & \text { if } \tau\left(R_{1}\right) \geq \frac{1}{2} \\
\max \left\{0,1-\tau\left(R_{1}\right)-\tau\left(R_{3}\right)\right\} & \text { if } \tau\left(R_{1}\right)<\frac{1}{2}\end{cases} \\
& \Psi_{3}(R)= \begin{cases}\min \left\{\tau\left(R_{3}\right), 1-\tau\left(R_{1}\right)\right\} & \text { if } \tau\left(R_{1}\right)<\frac{1}{2} \\
\max \left\{0,1-\tau\left(R_{1}\right)-\tau\left(R_{2}\right)\right\} & \text { if } \tau\left(R_{1}\right) \geq \frac{1}{2}\end{cases}
\end{aligned}
$$

Observe that $\Psi$ is tops-only. By an argument similar to the one used in Example 2 it is easy to see that $\Psi$ is bribe-proof. Consider any $R \in \mathcal{R}^{N}$ and $R_{1}^{\prime} \in \mathcal{R}$ such that $\left(\tau\left(R_{1}\right), \tau\left(R_{2}\right), \tau\left(R_{3}\right)\right)=\left(\frac{1}{4}, 1,1\right)$ and $\tau\left(R_{1}^{\prime}\right)=\frac{3}{4}$. Then, $\Psi(R)=\left(\frac{1}{4}, 0, \frac{3}{4}\right)$ and $\Psi\left(R_{1}^{\prime}, R_{2}, R_{3}\right)=\left(\frac{3}{4}, \frac{1}{4}, 0\right)$, which shows that $\Psi$ is not replacement monotonic.

In order to obtain a full characterization of all bribe-proof rules (by means of Pareto efficiency and strategy-proofness), we raise the following question: is there any weaker notion of replacement monotonicity that together with Pareto efficiency and strategy-proofness completely characterize the set of bribe-proof rules? Theorem 1 below answers the question in the affirmative and Definition 6 identifies precisely this weaker concept of replacement monotonicity.

Definition 5 A rule $\Phi$ is weakly replacement monotonic if for all $R \in \mathcal{R}^{N}$, all $i \in N$, and all $R_{i}^{\prime} \in \mathcal{R}$, if $\left[\Phi_{i}(R) \leq \Phi_{i}\left(R_{i}^{\prime}, R_{-i}\right)\right.$ and $\Phi_{i}(R) \neq \tau\left(R_{i}\right)$ or $\Phi_{i}\left(R_{i}^{\prime}, R_{-i}\right) \neq$ $\left.\tau\left(R_{i}^{\prime}\right)\right]$ then $\left[\Phi_{j}(R) \geq \Phi_{j}\left(R_{i}^{\prime}, R_{-i}\right)\right.$ for all $\left.j \neq i\right]$.

Remark 1 All replacement monotonic rules are weakly replacement monotonic.

Before stating Theorem 1 we want to point out the relationship between the two replacement monotonicity properties and the property of non-bossiness. A rule $\Phi$ is non-bossy if for all $R, R^{\prime} \in \mathcal{R}^{N}$ and $i \in N$ such that $R_{-i}=R_{-i}^{\prime}$, if $\Phi_{i}(R)=\Phi_{i}\left(R^{\prime}\right)$ then $\Phi(R)=\Phi\left(R^{\prime}\right)$. First, observe that replacement monotonicity implies nonbossiness. Second, Example 2 shows that weak replacement monotonicity does not 
imply non-bossiness ( $\psi$ is weakly replacement monotonic and bossy). However, weak replacement monotonicity and tops-onlyness imply non-bossiness.

Now, we are ready to state and prove our axiomatic characterization of bribeproof rules in terms of Pareto efficiency, strategy-proofness, and weak replacement monotonicity.

Theorem 1 A rule is bribe-proof if and only if it is Pareto efficient, strategy-proof, and weakly replacement monotonic.

\section{Proof of Theorem 1}

The following weaker notion of bribe-proofness will be useful in the proof of Theorem 1.

A rule $\Phi$ is individually bribe-proof if for all $R \in \mathcal{R}^{N}$, all $i \in N$, and all $R_{i}^{\prime} \in \mathcal{R}$ there are no $S \subseteq N$ and $\left(t_{j}\right)_{j \in S} \in \mathbb{R}^{S}$ such that $i \in S, \sum_{j \in S} t_{j}=\sum_{j \in S} \Phi_{j}\left(R_{i}^{\prime}, R_{-i}\right)$, $t_{j} R_{j} \Phi_{j}(R)$ for all $j \in S$, and $t_{i} P_{i} \Phi_{i}(R)$.

Observe that individually bribe-proof rules are immune to bribes in which the set of bribed agents is a singleton.

Remark 2 Assume $\Phi$ is bribe-proof. Then, $\Phi$ is individually bribe-proof.

Lemma 5 at the end of this section will show that both notions of bribe-proofness are indeed equivalent. The proof of Lemma 1 also shows that the following remark holds.

Remark 3 Let $\Phi$ be an individually bribe-proof rule. Then, $\Phi$ is Pareto efficient and strategy-proof.

The proof of Theorem 1 consists of three lemmata. Lemma 3 says that all Pareto efficient, strategy-proof, and weakly replacement monotonic rules are individually bribe-proof. Lemma 4 states that all individually bribe-proof rules are Pareto efficient, strategy-proof, and weakly replacement monotonic. Finally, Lemma 5 says that all individually bribe-proof rules are bribe-proof.

Lemma 3 Assume $\Phi$ is Pareto efficient, strategy-proof, and weakly replacement monotonic. Then, $\Phi$ is individually bribe-proof. 
Proof Let $\Phi$ be a Pareto efficient, strategy-proof, and weakly replacement monotonic rule. Assume that $\Phi$ is not individually bribe-proof; i.e., there exist $R \in \mathcal{R}^{N}$, $i \in S \subseteq N, R_{i}^{\prime} \in \mathcal{R}$ and $\left(t_{j}\right)_{j \in S} \in \mathbb{R}^{S}$ such that

$$
\sum_{j \in S} t_{j}=\sum_{j \in S} \Phi_{j}\left(R_{i}^{\prime}, R_{-i}\right)
$$

for all $j \in S$,

$$
t_{j} R_{j} \Phi_{j}(R)
$$

and

$$
t_{i} P_{i} \Phi_{i}(R)
$$

Since $\Phi$ is Pareto efficient,

$$
\Phi(R) \neq \Phi\left(R_{i}^{\prime}, R_{-i}\right) .
$$

Without loss of generality, we assume that $\sum_{j \in N} \tau\left(R_{j}\right) \geq 1$ (the proof when $\sum_{j \in N} \tau\left(R_{j}\right)<1$ is symmetric). By Pareto efficiency, $\Phi_{j}(R) \leq \tau\left(R_{j}\right)$ for all $j \in N$. By conditions (2) and (3),

$$
t_{j} \geq \Phi_{j}(R) \text { for all } j \in S
$$

and

$$
t_{i}>\Phi_{i}(R)
$$

By (1), (5), and (6),

$$
\sum_{j \in S} t_{j}=\sum_{j \in S} \Phi_{j}\left(R_{T}^{\prime}, R_{-T}\right)>\sum_{j \in S} \Phi_{j}(R)
$$

By $(3), \tau\left(R_{i}\right) \neq \Phi_{i}(R)$. Hence, by Lemma 2 , there exist $a\left(R_{-i}\right)$ and $b\left(R_{-i}\right)$ such that

$$
\Phi_{i}(R)=\operatorname{med}\left\{a\left(R_{-i}\right), b\left(R_{-i}\right), \tau\left(R_{i}\right)\right\}=b\left(R_{-i}\right) .
$$

By strategy-proofness of $\Phi, \Phi_{i}(R) R_{i} \Phi_{i}\left(R_{i}^{\prime}, R_{-i}\right)$.

- Assume $\Phi_{i}(R)=\Phi_{i}\left(R_{i}^{\prime}, R_{-i}\right)$. Because $\Phi_{i}(R)<\tau\left(R_{i}\right)$, by weak replacement monotonicity, $\Phi(R)=\Phi\left(R_{i}^{\prime}, R_{-i}\right)$, which contradicts condition (4).

- Assume $\Phi_{i}(R) \neq \Phi_{i}\left(R_{i}^{\prime}, R_{-i}\right)$. Because $\Phi_{i}(R)<\tau\left(R_{i}\right)$, by Lemma 2 applied to $R_{i}^{\prime}$ and condition $(8), \Phi_{i}\left(R_{i}^{\prime}, R_{-i}\right)=\operatorname{med}\left\{a\left(R_{-i}\right), b\left(R_{-i}\right), \tau\left(R_{i}^{\prime}\right)\right\} \leq b\left(R_{-i}\right)$. Thus, $\Phi_{i}(R)>\Phi_{i}\left(R_{i}^{\prime}, R_{-i}\right)$. Since $\Phi$ is weakly replacement monotonic,

$$
\Phi_{j}\left(R_{i}^{\prime}, R_{-i}\right) \geq \Phi_{j}(R) \text { for all } j \neq i .
$$


Then, $\sum_{j \in N} \Phi_{j}(R)=\sum_{j \in N} \Phi_{j}\left(R_{i}^{\prime}, R_{-i}\right)$, condition (9), and $i \in S$ imply $\sum_{j \notin S} \Phi_{j}\left(R_{i}^{\prime}, R_{-i}\right) \geq \sum_{j \notin S} \Phi_{j}(R)$. Thus, $\sum_{j \in S} \Phi_{j}(R) \geq \sum_{j \in S} \Phi_{j}\left(R_{i}^{\prime}, R_{-i}\right)=$ $\sum_{j \in S} t_{j}$, which contradicts condition $(7)$.

Lemma 4 Let $\Phi$ be an individually bribe-proof rule. Then, $\Phi$ is Pareto efficient, strategy-proof, and weakly replacement monotonic.

Proof Let $\Phi$ be an individually bribe-proof rule. Hence, by Remark 3, $\Phi$ is Pareto efficient and strategy-proof. Suppose $\Phi$ is not weakly replacement monotonic. Without loss of generality, assume there are $R \in \mathcal{R}^{N}, i \in N, R_{i}^{\prime} \in \mathcal{R}$ such that $\Phi_{i}\left(R_{i}^{\prime}, R_{-i}\right) \neq \tau\left(R_{i}^{\prime}\right)$, and either:

1. $\Phi_{i}(R)<\Phi_{i}\left(R_{i}^{\prime}, R_{-i}\right)$ and there exists $j \neq i$ such that $\Phi_{j}(R)<\Phi_{j}\left(R_{i}^{\prime}, R_{-i}\right)$, or

2. $\Phi_{i}(R)=\Phi_{i}\left(R_{i}^{\prime}, R_{-i}\right)$ and there exist $j, j^{\prime} \in N$ such that $\Phi_{j}(R)<\Phi_{j}\left(R_{i}^{\prime}, R_{-i}\right)$ and $\Phi_{j^{\prime}}\left(R_{i}^{\prime}, R_{-i}\right)<\Phi_{j^{\prime}}(R)$.

We will consider the two possibilities separately.

Case 1: Assume $\Phi_{i}(R)<\Phi_{i}\left(R_{i}^{\prime}, R_{-i}\right)$ and $\Phi_{j}(R)<\Phi_{j}\left(R_{i}^{\prime}, R_{-i}\right)$ for some $j \neq i$. We distinguish between two subcases:

Case 1.1: $\sum_{k \in N \backslash\{i\}} \tau\left(R_{k}\right)+\tau\left(R_{i}^{\prime}\right) \geq 1$.

By Pareto efficiency of $\Phi, \tau\left(R_{k}\right) \geq \Phi_{k}\left(R_{i}^{\prime}, R_{-i}\right)$ for every $k \in N \backslash\{i\}$ and $\tau\left(R_{i}^{\prime}\right)>\Phi_{i}\left(R_{i}^{\prime}, R_{-i}\right)$. Because $\tau\left(R_{j}\right) \geq \Phi_{j}\left(R_{i}^{\prime}, R_{-i}\right)>\Phi_{j}(R), \tau\left(R_{j}\right)>\Phi_{j}(R)$.

Therefore, by Pareto efficiency of $\Phi$,

$$
\tau\left(R_{k}\right) \geq \Phi_{k}(R) \text { for every } k \in N
$$

Let $S=\left\{k \in N \mid \Phi_{k}\left(R_{i}^{\prime}, R_{-i}\right)<\Phi_{k}(R)\right\} \cup\{i\}$. Note that, by feasibility, $S \neq\{i\}$ and because $\Phi_{j}(R)<\Phi_{j}\left(R_{i}^{\prime}, R_{-i}\right), j \notin S$. Since $\sum_{k \in N} \Phi_{k}\left(R_{i}^{\prime}, R_{-i}\right)=$ $\sum_{k \in N} \Phi_{k}(R)$ and $\sum_{k \notin S} \Phi_{k}\left(R_{i}^{\prime}, R_{-i}\right)>\sum_{k \notin S} \Phi_{k}(R), \quad \sum_{k \in S} \Phi_{k}\left(R_{i}^{\prime}, R_{-i}\right)<$ $\sum_{k \in S} \Phi_{k}(R)$. Therefore, by definition of $S$ and condition $(10), \Phi_{k}\left(R_{i}^{\prime}, R_{-i}\right)<$ $\Phi_{k}(R) \leq \tau\left(R_{k}\right)$ for all $k \in S \backslash\{i\}$, and by hypothesis, $\Phi_{i}(R)<\Phi_{i}\left(R_{i}^{\prime}, R_{-i}\right)<$ $\tau\left(R_{i}^{\prime}\right)$. Hence, there exists $\varepsilon>0$ sufficiently small such that

$$
\varepsilon<\sum_{k \in S} \Phi_{k}(R)-\sum_{k \in S} \Phi_{k}\left(R_{i}^{\prime}, R_{-i}\right)
$$

and $\varepsilon<\tau\left(R_{i}^{\prime}\right)-\Phi_{i}\left(R_{i}^{\prime}, R_{-i}\right)$. Condition (11) can be rewritten as,

$$
\Phi_{i}\left(R_{i}^{\prime}, R_{-i}\right)-\Phi_{i}(R)+\varepsilon<\sum_{k \in S \backslash\{i\}} \Phi_{k}(R)-\sum_{k \in S \backslash\{i\}} \Phi_{k}\left(R_{i}^{\prime}, R_{-i}\right) .
$$


For each $k \in S \backslash\{i\}$ there exists $\alpha_{k}>0$ such that $\alpha_{k}<\Phi_{k}(R)-\Phi_{k}\left(R_{i}^{\prime}, R_{-i}\right)$ and

$$
\sum_{k \in S \backslash\{i\}} \alpha_{k}=\Phi_{i}\left(R_{i}^{\prime}, R_{-i}\right)-\Phi_{i}(R)+\varepsilon .
$$

Define $t_{i}=\Phi_{i}\left(R_{i}^{\prime}, R_{-i}\right)+\varepsilon$ (or equivalently $t_{i}=\Phi_{i}(R)+\left[\Phi_{i}\left(R_{i}^{\prime}, R_{-i}\right)-\Phi_{i}(R)\right]+$ $\varepsilon)$ and $t_{k}=\Phi_{k}(R)-\alpha_{k}$ for all $k \in S \backslash\{i\}$. First, observe that, by definition of $t_{i}$ and $\left(t_{k}\right)_{k \in S \backslash\{i\}}, \Phi_{k}\left(R_{i}^{\prime}, R_{-i}\right)<t_{k}$ and $\Phi_{i}\left(R_{i}^{\prime}, R_{-i}\right)<t_{i}<\tau\left(R_{i}^{\prime}\right)$. Second,

$$
\begin{aligned}
\sum_{k \in S} t_{k} & =\sum_{k \in S \backslash\{i\}} \Phi_{k}(R)-\sum_{k \in S \backslash\{i\}} \alpha_{k}+\Phi_{i}(R)+\left[\Phi_{i}\left(R_{i}^{\prime}, R_{-i}\right)-\Phi_{i}(R)\right]+\varepsilon \\
& =\sum_{k \in S \backslash\{i\}} \Phi_{k}(R)-\Phi_{i}\left(R_{i}^{\prime}, R_{-i}\right)+\Phi_{i}(R)-\varepsilon+\Phi_{i}(R) \\
& =\sum_{k \in S} \Phi_{k}(R), \quad+\left[\Phi_{i}\left(R_{i}^{\prime}, R_{-i}\right)-\Phi_{i}(R)\right]+\varepsilon
\end{aligned}
$$

where the second equality follows from condition (12). Since $t_{k} P_{k} \Phi_{k}\left(R_{i}^{\prime}, R_{-i}\right)$ for all $k \in S$, it follows that $\Phi$ is not individually bribe-proof.

Case 1.2: $\sum_{k \in N \backslash\{i\}} \tau\left(R_{k}\right)+\tau\left(R_{i}^{\prime}\right)<1$.

By Pareto efficiency of $\Phi, \tau\left(R_{k}\right) \leq \Phi_{k}\left(R_{i}^{\prime}, R_{-i}\right)$ for every $k \in N \backslash\{i\}$ and $\tau\left(R_{i}^{\prime}\right) \leq \Phi_{i}\left(R_{i}^{\prime}, R_{-i}\right)$. But the hypothesis that $\Phi_{i}\left(R_{i}^{\prime}, R_{-i}\right) \neq \tau\left(R_{i}^{\prime}\right)$ implies $\tau\left(R_{i}^{\prime}\right)<\Phi_{i}\left(R_{i}^{\prime}, R_{-i}\right)$. By Lemma 2 ,

$$
\Phi_{i}\left(R_{i}^{\prime}, R_{-i}\right)=\operatorname{med}\left\{a\left(R_{-i}\right), b\left(R_{-i}\right), \tau\left(R_{i}^{\prime}\right)\right\}=a\left(R_{-i}\right)
$$

and

$$
\Phi_{i}(R)=\operatorname{med}\left\{a\left(R_{-i}\right), b\left(R_{-i}\right), \tau\left(R_{i}\right)\right\} \geq a\left(R_{-i}\right),
$$

implying $\Phi_{i}\left(R_{i}^{\prime}, R_{-i}\right) \leq \Phi_{i}(R)$, which contradicts the hypothesis that $\Phi_{i}(R)<$ $\Phi_{i}\left(R_{i}^{\prime}, R_{-i}\right)$.

Case 2: Assume $\Phi_{i}(R)=\Phi_{i}\left(R_{i}^{\prime}, R_{-i}\right)$ and there exist $j, j^{\prime} \in N$ such that $\Phi_{j}(R)<$ $\Phi_{j}\left(R_{i}^{\prime}, R_{-i}\right)$ and $\Phi_{j^{\prime}}\left(R_{i}^{\prime}, R_{-i}\right)<\Phi_{j^{\prime}}(R)$. We distinguish between two subcases: Case 2.1: $\sum_{k \in N \backslash\{i\}} \tau\left(R_{k}\right)+\tau\left(R_{i}^{\prime}\right) \geq 1$.

By Pareto efficiency of $\Phi, \Phi_{k}\left(R_{i}^{\prime}, R_{-i}\right) \leq \tau\left(R_{k}\right)$ for every $k \in N \backslash\{i\}$ and $\Phi_{i}\left(R_{i}^{\prime}, R_{-i}\right) \leq \tau\left(R_{i}^{\prime}\right)$. By assumption, $\Phi_{i}\left(R_{i}^{\prime}, R_{-i}\right) \neq \tau\left(R_{i}^{\prime}\right)$ and thus

$$
\Phi_{i}\left(R_{i}^{\prime}, R_{-i}\right)=\Phi_{i}(R)<\tau\left(R_{i}^{\prime}\right) .
$$

By Pareto efficiency of $\Phi$,

$$
\Phi_{j^{\prime}}\left(R_{i}^{\prime}, R_{-i}\right)<\Phi_{j^{\prime}}(R) \leq \tau\left(R_{j^{\prime}}\right) .
$$


By conditions (13) and (14), there exists $\varepsilon>0$ such that $\Phi_{i}\left(R_{i}^{\prime}, R_{-i}\right)<$ $\Phi_{i}(R)+\varepsilon<\tau\left(R_{i}^{\prime}\right)$ and $\Phi_{j^{\prime}}\left(R_{i}^{\prime}, R_{-i}\right)<\left(\Phi_{j^{\prime}}(R)-\varepsilon\right)<\Phi_{j^{\prime}}(R) \leq \tau\left(R_{j^{\prime}}\right)$. Since $P_{i}^{\prime}$ and $P_{j^{\prime}}$ are single-peaked preference relations, $\left(\Phi_{j^{\prime}}(R)-\varepsilon\right) P_{j^{\prime}} \Phi_{j^{\prime}}\left(R_{i}^{\prime}, R_{-i}\right)$ and $\left(\Phi_{i}(R)+\varepsilon\right) P_{i}^{\prime} \Phi_{i}\left(R_{i}^{\prime}, R_{-i}\right)$. Letting $S=\left\{i, j^{\prime}\right\}, t_{i}=\Phi_{i}(R)+\varepsilon$, and $t_{j^{\prime}}=$ $\left(\Phi_{j^{\prime}}(R)-\varepsilon\right)$ we conclude that $\Phi$ is not individually bribe-proof.

Case 2.2: $\sum_{k \in N \backslash\{i\}} \tau\left(R_{k}\right)+\tau\left(R_{i}^{\prime}\right)<1$.

By Pareto efficiency of $\Phi, \tau\left(R_{k}\right) \leq \Phi_{k}\left(R_{i}^{\prime}, R_{-i}\right)$ for every $k \in N \backslash\{i\}$ and $\tau\left(R_{i}^{\prime}\right) \leq \Phi_{i}\left(R_{i}^{\prime}, R_{-i}\right)$. But the hypothesis that $\Phi_{i}\left(R_{i}^{\prime}, R_{-i}\right) \neq \tau\left(R_{i}^{\prime}\right)$ implies

$$
\tau\left(R_{i}^{\prime}\right)<\Phi_{i}\left(R_{i}^{\prime}, R_{-i}\right)=\Phi_{i}(R)
$$

Since $\tau\left(R_{j^{\prime}}\right) \leq \Phi_{j^{\prime}}\left(R_{i}^{\prime}, R_{-i}\right)<\Phi_{j^{\prime}}(R)$ it follows, from the Pareto efficiency of $\Phi$, that $\sum_{k \in N} \tau\left(R_{k}\right) \leq 1$ and

$$
\tau\left(R_{j}\right) \leq \Phi_{j}(R)<\Phi_{j}\left(R_{i}^{\prime}, R_{-i}\right)
$$

By conditions (15) and (16), there exists $\varepsilon>0$ such that $\tau\left(R_{i}^{\prime}\right) \leq \Phi_{i}(R)-$ $\varepsilon<\Phi_{i}\left(R_{i}^{\prime}, R_{-i}\right)=\Phi_{i}(R)$ and $\tau\left(R_{j}\right)<\left(\Phi_{j}(R)+\varepsilon\right)<\Phi_{j}\left(R_{i}^{\prime}, R_{-i}\right)$. Since $P_{i}^{\prime}$ and $P_{j}$ are single-peaked preference relations, $\left(\Phi_{i}(R)-\varepsilon\right) P_{i}^{\prime} \Phi_{i}\left(R_{i}^{\prime}, R_{-i}\right)$ and $\left(\Phi_{j}(R)+\varepsilon\right) P_{j} \Phi_{j}\left(R_{i}^{\prime}, R_{-i}\right)$. Letting $S=\{i, j\}, t_{j}=\Phi_{j}(R)+\varepsilon$, and $t_{i}=$ $\left(\Phi_{i}(R)-\varepsilon\right)$ we conclude that $\Phi$ is not individually bribe-proof.

The proof of Theorem 1 finishes with Lemma 5 that, together with Remark 2, establishes the equivalence of the two notions of bribe-proofness.

Lemma 5 Assume $\Phi$ is individually bribe-proof. Then, $\Phi$ is a bribe-proof.

Proof Let $\Phi$ be an individually bribe-proof rule and assume that $\Phi$ is not bribeproof; namely, there exist $R \in \mathcal{R}^{N}, T \subseteq S \subseteq N$ with $\# T \geq 2, R_{T}^{\prime} \in \mathcal{R}^{T}$, and $\left(t_{j}\right)_{j \in S} \in \mathbb{R}^{S}$ such that $\sum_{j \in S} t_{j}=\sum_{j \in S} \Phi_{j}\left(R_{T}^{\prime}, R_{-T}\right)$, for all $j \in S$,

$$
t_{j} R_{j} \Phi_{j}(R)
$$

and for all $i \in T$,

$$
t_{i} P_{i} \Phi_{i}(R) .
$$

Without loss of generality, assume that $T$ is minimal in the sense that for all $i \in T$ there are no $\bar{S} \supseteq T \backslash\{i\}, \bar{R}_{T \backslash\{i\}} \in \mathcal{R}^{T \backslash\{i\}}$, and $\left(\bar{t}_{j}\right)_{j \in \bar{S}} \in \mathbb{R}^{\bar{S}}$ with the property that $\sum_{j \in \bar{S}} \bar{t}_{j}=\sum_{j \in \bar{S}} \Phi_{j}\left(\bar{R}_{T \backslash\{i\}}, R_{-T \backslash\{i\}}\right), \bar{t}_{j} R_{j} \Phi_{j}(R)$ for all $j \in \bar{S}$, and $\bar{t}_{\hat{\imath}} P_{\hat{\imath}} \Phi_{\hat{\imath}}(R)$ for all $\hat{\imath} \in T \backslash\{i\}$. Assume that $\sum_{j \in N} \tau\left(R_{j}\right) \geq 1$ (the other case is done symmetrically). By 
Remark 3, $\Phi$ is Pareto efficient. Then, (17), (18), and $\sum_{j \in S} t_{j}=\sum_{j \in S} \Phi_{j}\left(R_{T}^{\prime}, R_{-T}\right)$ imply

$$
\sum_{j \in S} \Phi_{j}\left(R_{T}^{\prime}, R_{-T}\right)>\sum_{j \in S} \Phi_{j}(R)
$$

We claim that by the minimality condition of the set $T$, for each $i \in T$,

$$
\sum_{j \in S} \Phi_{j}\left(R_{T \backslash\{i\}}^{\prime}, R_{i}, R_{-T}\right) \leq \sum_{j \in S} \Phi_{j}(R)
$$

Assume otherwise; i.e., there exists $i \in T$ such that

$$
\sum_{j \in S} \Phi_{j}(R)<\sum_{j \in S} \Phi_{j}\left(R_{T \backslash\{i\}}^{\prime}, R_{i}, R_{-T}\right) .
$$

Pareto efficiency of $\Phi, \sum_{j \in N} \tau\left(R_{j}\right) \geq 1$, and (18) imply that for all $k \in T \backslash\{i\}$, $\tau\left(R_{k}\right)>\Phi_{k}(R)$. Take $k \in T \backslash\{i\}$ and consider any $\bar{R}_{k} \in \mathcal{R}$ such that $\tau\left(\bar{R}_{k}\right)=1$. Observe that, by Remark $3, \Phi$ is strategy-proof. Hence, by Lemma $2, \Phi_{k}(R)=$ $\Phi_{k}\left(\bar{R}_{k}, R_{-k}\right)$. By Lemma 4 , and since $\Phi$ is individually bribe-proof, $\Phi$ is weakly replacement monotonic. Thus,

$$
\Phi\left(\bar{R}_{k}, R_{-k}\right)=\Phi(R)
$$

Hence,

$$
\begin{aligned}
\sum_{j \in S} \Phi_{j}\left(\bar{R}_{k}, R_{-k}\right) & =\sum_{j \in S} \Phi_{j}(R) \\
& <\sum_{j \in S} \Phi_{j}\left(R_{T \backslash\{i\}}^{\prime}, R_{i}, R_{-T}\right) \quad \text { by }(21) \\
& \leq \sum_{j \in S \backslash\{k\}} \tau\left(R_{j}\right)+\tau\left(\bar{R}_{k}\right) \quad \text { since } \tau\left(\bar{R}_{k}\right)=1 .
\end{aligned}
$$

Since $\tau\left(\bar{R}_{k}\right)=1$, there exists $\left(\bar{t}_{j}\right)_{j \in S} \in \mathbb{R}^{S}$ such that $\sum_{j \in S} \bar{t}_{j}=\sum_{j \in S} \Phi_{j}\left(R_{T \backslash\{i\}}^{\prime}, R_{i}, R_{-T}\right)$, and for all $j \in S, \Phi_{j}(R)=\Phi_{j}\left(\bar{R}_{k}, R_{-k}\right)<\bar{t}_{j} \leq \tau\left(R_{j}\right)$. Hence, for all $j \in S$, $\bar{t}_{j} P_{j} \Phi_{j}(R)$, contradicting the minimality of $T$ because $S$ would bribe $T \backslash\{i\}$ at $R$ through $R_{T \backslash\{i\}}^{\prime}$. Thus, (20) holds.

From (19) and (20), we obtain

$$
\sum_{j \in S} \Phi_{j}\left(R_{T \backslash\{i\}}^{\prime}, R_{i}, R_{-T}\right)<\sum_{j \in S} \Phi_{j}\left(R_{T}^{\prime}, R_{-T}\right)=\sum_{j \in S} t_{j}
$$

implying that the set $S$ bribes agent $i$ at profile $\left(R_{T \backslash\{i\}}^{\prime}, R_{i}, R_{-T}\right)$, contradicting the assumption that $\Phi$ was individually bribe-proof. 


\section{Final Remarks}

Before finishing the paper, three remarks about the definition of bribe-proofness are appropriate. First, and as it has already been said, Schummer (2000) defines a bribe by requiring that the set of bribed agents $T$ as well as $S \backslash T$ be, at most, singleton. If we would ask for this additional requirement our class of bribe-proof rules would be substantially enlarged since there would be many bribe-proof rules without being weakly replacement monotonic. To see this, consider the case where $N=\{1,2,3,4\}$ and $R \in \mathcal{R}^{N}$ is such that $\Phi_{1}(R)<\tau\left(R_{1}\right){ }^{5} \quad$ Let $R_{1}^{\prime} \in \mathcal{R}$ be such that $\tau\left(R_{1}^{\prime}\right)=\Phi_{1}(R)-\varepsilon$ and assume $\Phi_{2}\left(R_{1}^{\prime}, R_{-1}\right)=\Phi_{2}(R)+\frac{3}{4} \varepsilon<\tau\left(R_{2}\right)$, $\Phi_{3}\left(R_{1}^{\prime}, R_{-1}\right)=\Phi_{3}(R)+\frac{3}{4} \varepsilon<\tau\left(R_{3}\right)$, and $\Phi_{4}\left(R_{1}^{\prime}, R_{-1}\right)=\Phi_{4}(R)-\frac{1}{2} \varepsilon$. Observe that $\Phi$ is not weakly replacement monotonic. Moreover, $\Phi$ is not bribe-proof (according to our definition) because there exist $S=\{1,2,3\}, t_{1}=\Phi_{1}(R)+\frac{\varepsilon}{6}, t_{2}=\Phi_{2}(R)+\frac{\varepsilon}{6}$, and $\Phi_{3}(R)+\frac{\varepsilon}{6}$ such that $t_{1}+t_{2}+t_{3}=\sum_{j \in S} \Phi_{j}\left(R_{1}^{\prime}, R_{-1}\right)$ and $t_{j} P_{j} \Phi_{j}(R)$ for all $j \in S$. However, $\Phi$ would be bribe-proof in the sense of Schummer (2000) because neither agent 2 nor agent 3 alone can compensate agent 1 because with agent 1's misrepresentation they gain $\frac{3}{4} \varepsilon$ while agent 1 loss is $\varepsilon$.

Second, our definition of bribe-proofness requires that all bribed agents should be strictly better off after the bribe. If instead we admit that a bribed agent be indifferent after the misrepresentation and the reallocation then bribe-proofness would be too restrictive. For instance, the rules of Examples 1, 2, and 3 are no longer bribe-proof in this stronger sense. Moreover, the uniform rule $U$ does not satisfy this stronger notion of bribe-proofness. To see that let $N=\{1,2,3\}$ be the set of agents and consider any $R=\left(R_{1}, R_{2}, R_{3}\right) \in \mathcal{R}^{N}$ such that $\tau\left(R_{1}\right)=\tau\left(R_{2}\right)=1$ and $\tau\left(R_{3}\right)=0$. Then, $U(R)=\left(\frac{1}{2}, \frac{1}{2}, 0\right)$. Consider now any $\left(R_{2}^{\prime}, R_{3}^{\prime}\right) \in \mathcal{R}^{\{2,3\}}$ such that $\tau\left(R_{2}^{\prime}\right)=\frac{3}{4}$ and $\tau\left(R_{3}^{\prime}\right)=1$. Then, $U\left(R_{1}, R_{2}^{\prime}, R_{3}^{\prime}\right)=\left(\frac{1}{3}, \frac{1}{3}, \frac{1}{3}\right)$. Let $T=S=\{2,3\}$ and $t_{2}=\frac{2}{3}$ and $t_{3}=0$. Since $t_{2}=\frac{2}{3} P_{2} \frac{1}{2}=U(R)$ and $t_{3}=0 R_{3} 0=U_{3}(R)$. Thus, $U$ is not bribe-proof in this stronger sense. All sequential dictator rules (roughly, given an order of agents, they receive sequentially their tops, up to feasibility) are bribe-proof in this stronger sense. We conjecture that this set of rules (one for each

\footnotetext{
${ }^{5}$ In order to make the argument more transparent and brief we omit the complete (and tedious) description of $\Phi$. Roughly, $\Phi$ is defined as follows: for all $R \in \mathcal{R}^{N}$ such that $\sum_{j \in N} \tau\left(R_{j}\right)<1$ or $\tau\left(R_{1}\right) \geq \frac{1}{4}, \Phi(R)=U(R)$, where $U$ is the uniform rule, while for all $R \in \mathcal{R}^{N}$ such that $\sum_{j \in N} \tau\left(R_{j}\right) \geq 1$ and $\tau\left(R_{1}\right)<\frac{1}{4}, \Phi_{1}(R)=\tau\left(R_{1}\right)$ and the remaining $1-\tau\left(R_{1}\right)$ is split among agents 2,3 , and 4 according to a weakly sequential rule (see Massó and Neme (2003)) defined by the function $g$ (for $N^{\prime}=\{2,3,4\}$ ) associated to the uniform rule relative to $q^{H}=q^{L}=$ $\left(\frac{3-4 \tau\left(R_{1}\right)+4 \varepsilon}{8}, \frac{3-4 \tau\left(R_{1}\right)+4 \varepsilon}{8}, \frac{1}{4}-\varepsilon\right)$ for a given $\varepsilon>0$ such that $\varepsilon<\frac{1}{4}-\tau\left(R_{1}\right)$.
} 
order) coincides with the class of rules that satisfy bribe-proofness in this stronger sense.

Third, in Massó and Neme (2003) we present a complete description of the full class of bribe-proof and tops-only rules by weakening condition (4.) in the description of sequential allotment rules (page 13 in Barberà, Jackson, and Neme (1997)). This class, the set of weakly sequential rules, can be described by using their definition but replacing condition (4.) by the following condition:

Let $q^{n}=g^{n}\left(q^{0}, R\right)$ and $q^{\prime n}=g^{n}\left(q^{0},\left(R_{i}^{\prime}, R_{-i}\right)\right)$. Then,

if $\tau\left(R_{i}^{\prime}\right)<\tau\left(R_{i}\right)$ and $\sum_{i \in N} \tau\left(R_{i}\right) \geq 1$ then $q_{j}^{\prime n} \geq q_{j}^{n}$ for every $j \neq i$;

if $\tau\left(R_{i}^{\prime}\right)>\tau\left(R_{i}\right)$ and $\sum_{i \in N} \tau\left(R_{i}\right)<1$ then $q_{j}^{\prime n} \leq q_{j}^{n}$ for every $j \neq i$.

\section{References}

[1] Barberà, S.: "Notes on strategy-proof social choice functions," in Social Choice Re-examinated, Arrow, K., Sen, A., and Suzumura, K. editors, Mac Millan (1996). French version: "Sur les fonctions de choix non manipulables," Revue d'Économie Politique 106, 61-81 (1996).

[2] Barberà, S.: "An introduction to strategy-proof social choice functions," Social Choice and Welfare 18, 619-653 (2001).

[3] Barberà, S.: "Strategy-proof Social Choice," mimeo (2006).

[4] Barberà, S., Jackson, M., and Neme, A.: "Strategy-proof allotment rules," Games and Economic Behavior 18, 1-21 (1997).

[5] Esö, P. and Schummer, J.: "Bribing and signalling in second price auctions," Games and Economic Behavior 47, 299-324 (2004).

[6] Fiestras-Janeiro, G., Klijn, F., and Sánchez, E.: "Manipulation of Optimal Matchings via Predonation of Endowment," Mathematical Social Sciences 47, 295-312 (2004).

[7] Massó, J. and Neme, A.: "Bribe-proof rules in the division problem," UFAE and IAE Working Papers 571.03 (2003).

[8] Moulin, H.: "On strategy-proofness and single-peakedness," Public Choice 35, 437-455 (1980). 
[9] Schummer, J.: "Manipulation through bribes," Journal of Economic Theory 91, 180-198 (2000).

[10] Sprumont, Y.: "The division problem with single-peaked preferences: a characterization of the uniform allocation rule," Econometrica 59, 509-519 (1991).

[11] Sprumont, Y.: "Strategy-proof collective choice in economic and political environments," Canadian Journal of Economics 28, 68-107 (1995). 